\title{
Article
}

\section{Is the Internet really ruining your memory? Keep reading as modifiable protective factor against cognitive impairment}

\section{Hernán Ramos ${ }^{1,2}$, Mónica Alacreu ${ }^{3}, \mathbf{M}^{\mathrm{a}}$ Dolores Guerrero² ${ }^{2}$, Rafael Sánchez ${ }^{4}$, Lucrecia Moreno $^{2 *}$}

${ }^{1}$ Community Pharmacist, Official College of Pharmacists of Valencia, Spain. ramgarher@alumnos.uchceu.es

${ }^{2}$ Department of Pharmacy, Universidad CEU Cardenal Herrera, Valencia, Spain. ma_dolores.guerrero@uchceu.es

${ }^{3}$ Embedded Systems and Artificial Intelligence Group, Universidad CEU Cardenal Herrera, Valencia, Spain. monica.alacreu@uchceu.es

${ }^{4}$ Neurology Service, Arnau de Vilanova Hospital, Valencia, Spain. rafael.sanchez2@uchceu.es

* Correspondence: Prof. Lucrecia Moreno Royo. 1moreno@uchceu.es

\begin{abstract}
:
Background: Subjective cognitive decline (SCD) would correspond to a preclinical phase of Alzheimer's disease. The aim of this study was to find associations between lifestyle individual factors compatible scores with cognitive impairment (CI) in SCD people.

Methods: This is a case-control study to detect SCD, CI and potential associated factors in 497 patients over 50 years in Community Pharmacies. Three screening tests detected possible CI and patients with at least one test compatible with CI were referred to Primary Care to be evaluated.

Results: In self-complaint patients statistically significant with depressive feelings were found $(86.8 \%)$ with benzodiazepines consumers $(83.9 \%)$ and female patients $(81.2 \%)$. Thirty percent of our sample obtained scores compatible with CI. Being older than 70 years old increased the odds of obtaining scores compatible with CI. High level education, reading and internet use were able to reduce the odds of positive scores compatible with CI (37\%-91\%, 7\%-18\% and $67 \%-86 \%)$, whereas, one extra hour television/day increased the risk in $8 \%-30 \%$. Reading was able to nullify the effect of both internet and TV consumption.
\end{abstract}

Conclusion: Not just the age but also modifiable lifestyle factors are acting in favour of a cognitive decline.

Keywords: cognitive impairment screening; cognitive reserve; subjective memory complaints; Internet; television; reading; marital status; sleep.

\section{Introduction}

Alzheimer's disease is the most common cause of dementia among older adults. It is an irreversible, progressive brain disorder, which slowly destroys memory, thinking skills and, eventually, the ability to carry out the simplest tasks. Related to age, little more is known about its aetiology however, it is known that that modifiable lifestyle could control a person's risk of 
developing the condition [1]. Therefore, knowledge about risk factors allows recognizing early symptoms for early detection of Alzheimer's disease [2].

Mild Cognitive Impairment (MCI) is an intermediate stage between normal cognitive deterioration due to aging and more serious deterioration of Alzheimer's dementia. It can involve problems with memory, language, thinking and judgment that are more serious than the normal changes related to aging. The prevalence in Spain is $9.6 \%$, it affects more the elderly and the female [3]. Since the description of mild cognitive impairment (CI) as a clinical entity, there has been great interest in identifying at the earliest, individuals at the preclinical stage of the disease.

Currently, a stage has been proposed prior to the known MCI. This stage, known as subjective cognitive decline (SCD), would correspond to this preclinical phase of Alzheimer's disease in some individuals. Two criteria define SCD. Firstly, a persistent decline in cognitive ability experienced by the patient, compared with a previous normal cognitive state, which is unrelated to an acute event. Second, correct performance on the standardised cognitive tests used to classify MCI [4].

It has been described that people with SCD are at increased risk of future cognitive impairment and dementia, in fact SCD may be the first symptom of early neurodegenerative disease [5]. These data support the importance of exploring the potential role of the disease as an early sign of neurodegenerative disorders [4].

Moreover, differences in the trajectories of cognitive aging have been observed since there are cases of older people with considerable cerebral pathology without exhibiting concomitant decreases in cognition. Therefore, there does not appear to be a direct relationship between the degree of brain pathology or brain damage and the clinical manifestation of that damage. This discrepancy is explained by the theory of cognitive reserve and the variations in cognitive aging by proposing that people with greater cognitive reserve may allow being more resilient than others and will show more belatedly the corresponding clinic [6].

Cognitive reserve is determined by genetic and neurodevelopmental factors although it may vary depending on the environment and exposure to certain factors such as education and lifestyle. Therefore, it is considered that the cognitive reserve is the result of an interaction between the genetics, the environment and the experiences of the person and that it can be transformed into a set of skills that allows to actively compensating for the effects of the disease. However, cognitive reserve is still a hypothetical construct that can only be measured indirectly by proxy indicators considered representative [7].

An active cognitive lifestyle was associated with protection from cerebrovascular disease. Protective lifestyle factors have been identified that may contribute to increased cognitive reserve, such as social engagement and cognitive stimulation. 
Good social connections may also increase cognitive reserve and protect against declining cognitive function. However, the association between social connections and cognitive function is less clear as poor social relationships may be a consequence of cognitive decline rather than a cause. Having fewer social contacts, a smaller social network and less engagement in social activity are associated with worst cognitive function [8].

Nowadays, Internet use has reinvented the ways in which we manage our social networks and relationships. Moreover, with the advent of smartphones, Internet access has become portable. The widespread use of Internet has introduced the opportunity to learn and to interact with society; even simple interactions through the smartphone's touchscreen interface have been demonstrated neural changes in cortical regions associated with sensory and motor processing of the hand and thumb [9].

Meanwhile, digital distractions on the Internet seem to create a non-ideal environment for the refinement of higher cognitive functions during critical periods of brain development in children and adolescents. On the other hand, the opposite may be true in older adults who experience cognitive impairment, for whom the online environment may provide a new source of positive cognitive stimulation [10].

The aim of this study is to investigate the association between modifiable protective/risk factors such as education, lifestyle and cognitive impairment in patients with subjective memory complaints (SMC) in order to develop cognitive effective cognitive training programs.

\section{Experimental Section}

The next research is part of a multidisciplinary project elaborated by the CRIDECO group, formed by professionals of different disciplines: community pharmacists, family practitioners, neurologists, psychologists and mathematicians. An analytical, longitudinal, observational, retrospective casecontrol study was planned to detect SCD, CI and potential associated factors in Community Pharmacy. The target population was people aged 50 and older with SMC.

\subsection{Patients recruitment.}

As shown in Figure 1, the initial number of people screened was 502. Definitely, 497 people met the inclusion criteria: being 50 years or older, having subjective memory complaints and accepting the informed consent of the study. The other five people were discarded for meeting any of the exclusion criteria: having a diagnosis of dementia or a severe physical or sensory deficit. Community pharmacist, previously trained, performs an active screening to detect cognitive alterations among individuals aged 50 and over who come to the community pharmacy. 


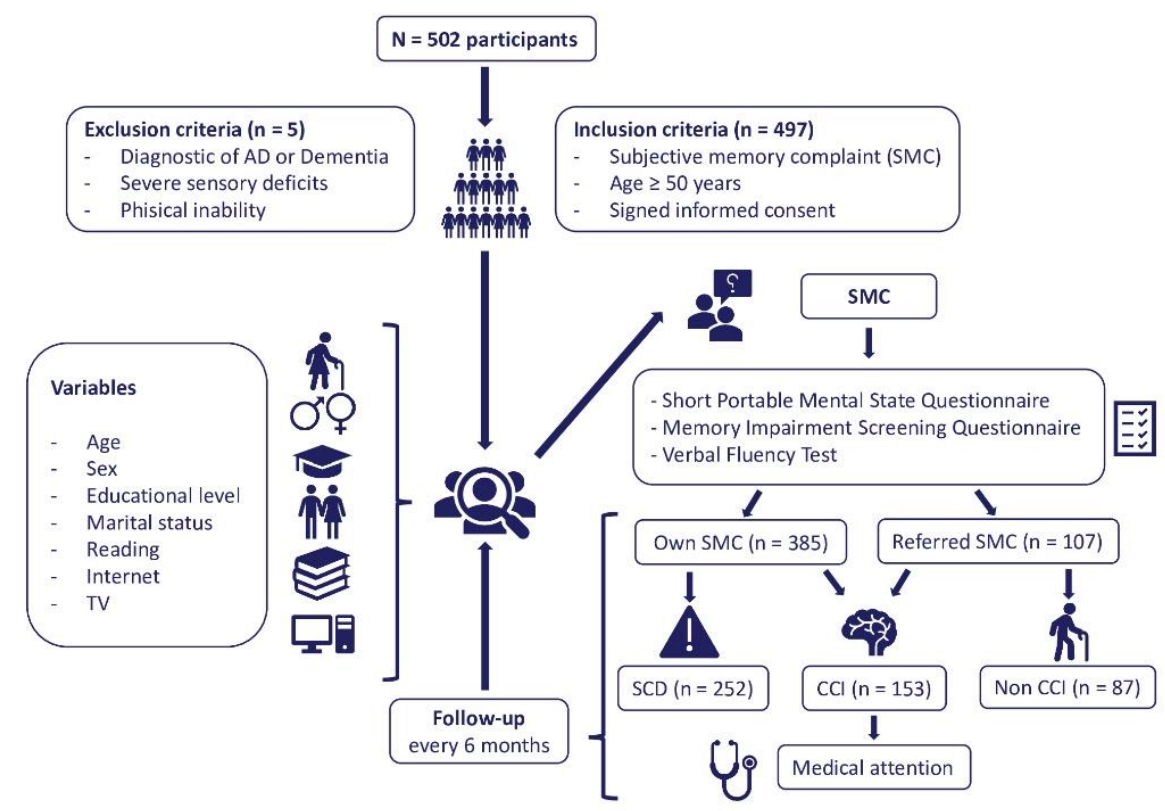

Figure 1. Methodological diagram of the analysis. AD: Alzheimer Disease, SMC: subjective memory complaint, SCD: subjective cognitive decline, CCI: compatible cognitive impairment.

The pharmacist during the dispensing detect the SMC (by express reference of the patient or by indirect question of referred by others), appearance of depressive feelings, increase of drowsiness, alteration in the recognition of objects, alteration in the language, difficulty to perform some complex activities such as using public transport, managing money and / or following medical treatment. Patient identified with SMC were informed of the study information and Signed Consent be requested.

\subsection{Data collection.}

Three validated screening tests be completed with all the study variables in order to collect the largest number of factors related to CI. Specifically: Memory Impairment Screening (MIS) [11], Short Portable Mental State Questionnaire (SPMSQ) [12] and Semantic Verbal Fluency (FVS) [13]. The tests used have been chosen after consultation with the Valencian Society of SM.

Patients with a score, in any of the 3 tests used for screening, compatible with the presence of cognitive impairment (CCI) will be referred to Primary Care for diagnosis. Patients with correct test performance and own SMC are classified with SCD and will continue to be followed up specially, as this would be a high-risk group (Figure 1). Finally, patients with correct test performance and referred SMC are classified as not compatible with cognitive impairment (Non CCI). All patients are followed up every 6 months, where they are re-evaluated by the three screening tests.

For the collection of information on the possible associated variables in the detected cases of possible $\mathrm{CI}$, a questionnaire was designed specifically by our research team. This includes all the study variables in order to collect the greatest number of factors related to $\mathrm{CI}$ that have been found in the consulted bibliography: demographic and lifestyle factors. 
The demographic variables measured are sex, age, weight, family history of cognitive impairment, education level, own or referred SMC, marital status and depressive feelings. On the other hand, the variables measured with respect to lifestyle are physical exercise, pastime, reading, time spent sleeping overnight and during the day, memory training, puzzles, games, Internet and TV consumption time. About lifestyle factors, we asked about the frequency: same as before, more or less than before perceiving SMC. The extensive fieldwork was carried out from September 2018 to January 2020.

\subsection{Sample size calculation}

This sample size is larger than necessary (385 people) to estimate the prevalence of people with a preliminary assessment compatible with $\mathrm{CI}$, under $\mathrm{SMC}$, assumed to be $50 \%$ (statistically more unfavourable situation), with an accuracy of $10 \%$ and a confidence level of $95 \%$.

\subsection{Statistical treatment of data}

All patient information is collected on paper as a medical history. A single responsible person is in charge of keeping the database updated, dumping all the information obtained from each patient in an Excel spreadsheet, designed specifically for this purpose. The statistical analysis of the data has been carried out using the advanced statistical software " $\mathrm{R}$ ". The prevalence of people with a CI compatible score, under SMC, has been estimated with a 95\% confidence. The association of patients obtaining scores compatible with $\mathrm{CI}$ with respect to the variables that determine their profile and with respect to the variables on their living and intellectual habits has been analyzed. Likewise, the association between profile variables and habits has been analyzed.

To determine the association between qualitative variables, the Chi-square test and the Fisher's exact test were used. To analyze the association with respect to quantitative variables, the following tests have been used: Pearson's test of independence on the correlation coefficient, Student's T test for independent samples, ANOVA and Kruskal-Wallis. Finally, in order to determine the protective or risk effect of the variables, as well as to quantify their effect, both univariate and multivariate logistic regression models have been adjusted, obtaining estimates of the odds ratio (OR), with 95\% confidence.

\subsection{Ethical approval.}

The study was reviewed and approved by the Research Ethics Committee of Universidad CEU Cardenal Herrera (approval no. CEI18/027) and by the Research Ethics Committee with drugs of Arnau de Vilanova Hospital (MOR-ROY-2018-013). All subjects gave written informed consent in accordance with the Declaration of Helsinki.

\section{Results}

\subsection{Subjective cognitive complaint patient's population characteristics}

The following results are based on a sample of 497 patients over 50 years of age, who were screened in one of the 19 collaborating pharmacies. These patients were included due to subjective memory complaints and expressly consented to participate in the study. 
According to SMC type, patients of the study were classified into 2 groups: patients with own subjective complaint or referred subjective complaint regarding if they were referred by himself or by a family member or the pharmacist, respectively. This classification has been used as one of the non-modifiable characteristics that define the profile of the patients, such as sex, age, among others.

Regarding if to SMC type is related to any other characteristics of the patient profile, statistically significantly differences were observed between genders, being more prevalent in women than in men $(81.2 \%$ vs $70.2 \%$, respectively, p-value $<0.05)$. In addition, this point is significantly more frequent in patients who feel depressed than in patients who do not feel depressed $(86.8 \%$ vs $74.5 \%$, respectively, $\mathrm{p}$-value $<0.05)$. Moreover, the subjective self-complaint is significantly more frequent in patients who are medicated with benzodiazepines, than in patients who are not medicated with benzodiazepines $(83.9 \%$ vs $73.1 \%$, respectively, p-value $<0.05)$. Similarly, referred subjective complaint is significantly more frequent in men, in patients who do not feel depressed or in patients who do not take benzodiazepines ( $p$-value $<0.05$ ). Conversely, no statistically significant differences were observed regarding subjective self-reported or referred memory complaint, with age, family history of CI, education or marital status.

\subsection{Patients scores compatible with cognitive impairment}

After screening, patients were classified into two groups: patients with scores compatible with $\mathrm{CI}$, on one of the three questionnaires used, or patients with scores incompatible with $\mathrm{CI}$, on all questionnaires (Table 1). This classification determines the variable of interest in our research, because it allows us to identify which patients are susceptible to being neurologically examined.

According to Table 1, 30.8\% of patients have obtained scores compatible with CI. This means, with $95 \%$ confidence that between $26.9 \%$ and $35 \%$ of patients over 50 with subjective memory complaints in the general population would obtain scores compatible with CI.

Table 1: Classification of patients into scores that are incompatible and compatible with suffering from $\mathrm{CI}$, according to the different screening questionnaires. CI: cognitive impairment.

\begin{tabular}{|c|c|c|c|c|}
\hline Test & Total & $\begin{array}{l}\text { Patients with scores } \\
\text { incompatible with CI }\end{array}$ & $\begin{array}{l}\text { Patients with scores } \\
\text { compatible with CI }\end{array}$ & Total \\
\hline & $\mathrm{n}(\%$ column $)$ & $\mathrm{n}(\%$ row $)$ & $\mathrm{n}(\%$ row $)$ & $\mathrm{n}(\%$ row $)$ \\
\hline \multicolumn{5}{|l|}{ SPMSQ } \\
\hline 1.Normal & $400(80.4 \%)$ & $344(86 \%)$ & $56(14 \%)$ & $400(100 \%)$ \\
\hline 2.Slight Impairment & $74(14.9 \%)$ & $0(0 \%)$ & $74(100 \%)$ & $74(100 \%)$ \\
\hline 3.Moderate impairment & $19(3.8 \%)$ & $0(0 \%)$ & $19(100 \%)$ & $19(100 \%)$ \\
\hline 4.Severe impairment & $4(0.8 \%)$ & $0(0 \%)$ & $4(100 \%)$ & $4(100 \%)$ \\
\hline \multicolumn{5}{|l|}{ MIS Questionnaire } \\
\hline 1.Normal & $412(82.9 \%)$ & $344(83.5 \%)$ & $68(16.5 \%)$ & $412(100 \%)$ \\
\hline 2.Impairment & $85(17.1 \%)$ & $0(0 \%)$ & $85(100 \%)$ & $85(100 \%)$ \\
\hline \multicolumn{5}{|l|}{ Verbal Fluency Questionnaire } \\
\hline 1.Normal & $423(85.1 \%)$ & $344(83.3 \%)$ & 79 (18.7\%) & $423(100 \%)$ \\
\hline
\end{tabular}




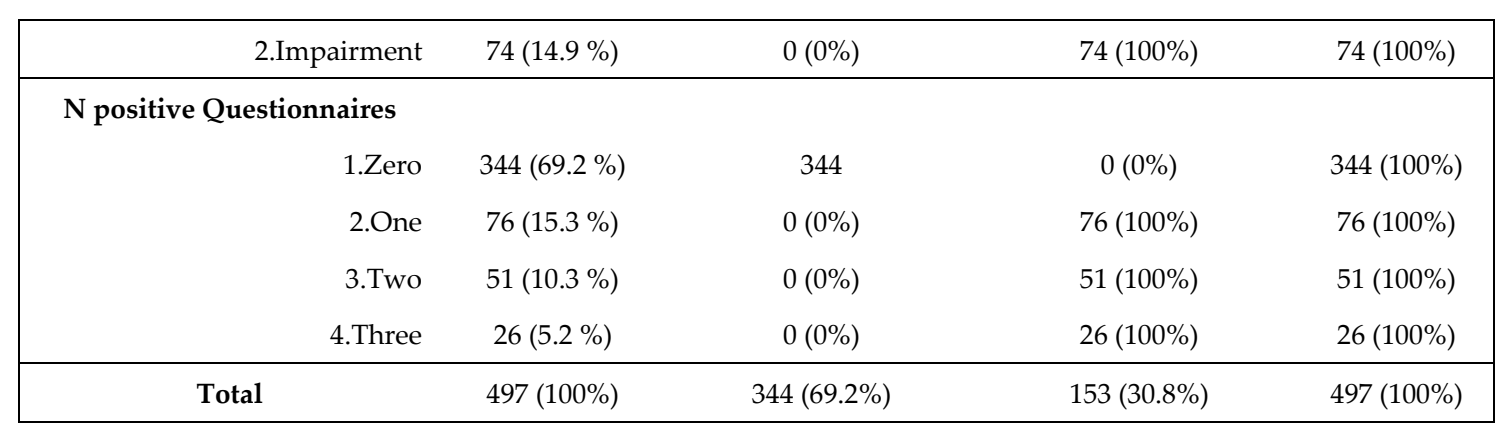

The association of obtaining scores compatible with CI and the characteristics that define the patient profile have been investigated (Table 2). The association with variables that define living and intellectual habits has also been analyzed (Tables 3 and 4). The three tables show the p-values of association between the analyzed characteristic and obtaining scores compatible with $\mathrm{CI}$ in the tests. In case of association, the OR by univariate logistic regression has been estimated with 95\% confidence. For qualitative variables, the OR estimate for each category is given with respect to the reference category, indicated by 1 .

Regarding the type of SMC there is no significant association with sex, family history of CI and depression. However, patient age, type of SMC, educational level and marital status do show an association with obtaining scores compatible with CI.

Specifically, it is estimated that being 70-79 years old can increase the odds of obtaining CIcompatible scores by 2.24-10.33 times for younger patients, aged 50-59. This rate is the highest among the characteristics that determine the profile of patients. On the other hand, the subjective complaint of own memory presents an OR ranging from 1.35 to 3.90. This data is almost 4 times higher than the referred memory complaint.

Table 2: Association between obtaining or not obtaining scores compatible with $\mathrm{CI}$ and the variables on the patient profile. a: Chi-square test; b: Fisher exact test (univariate logistic models: *: p-value $<0.1$; ${ }^{* *}$ : p-value $<0.05 ;{ }^{* * *}$ : p-value $<0.001$.

CI: cognitive impairment. SMC: subjective memory complaint. 


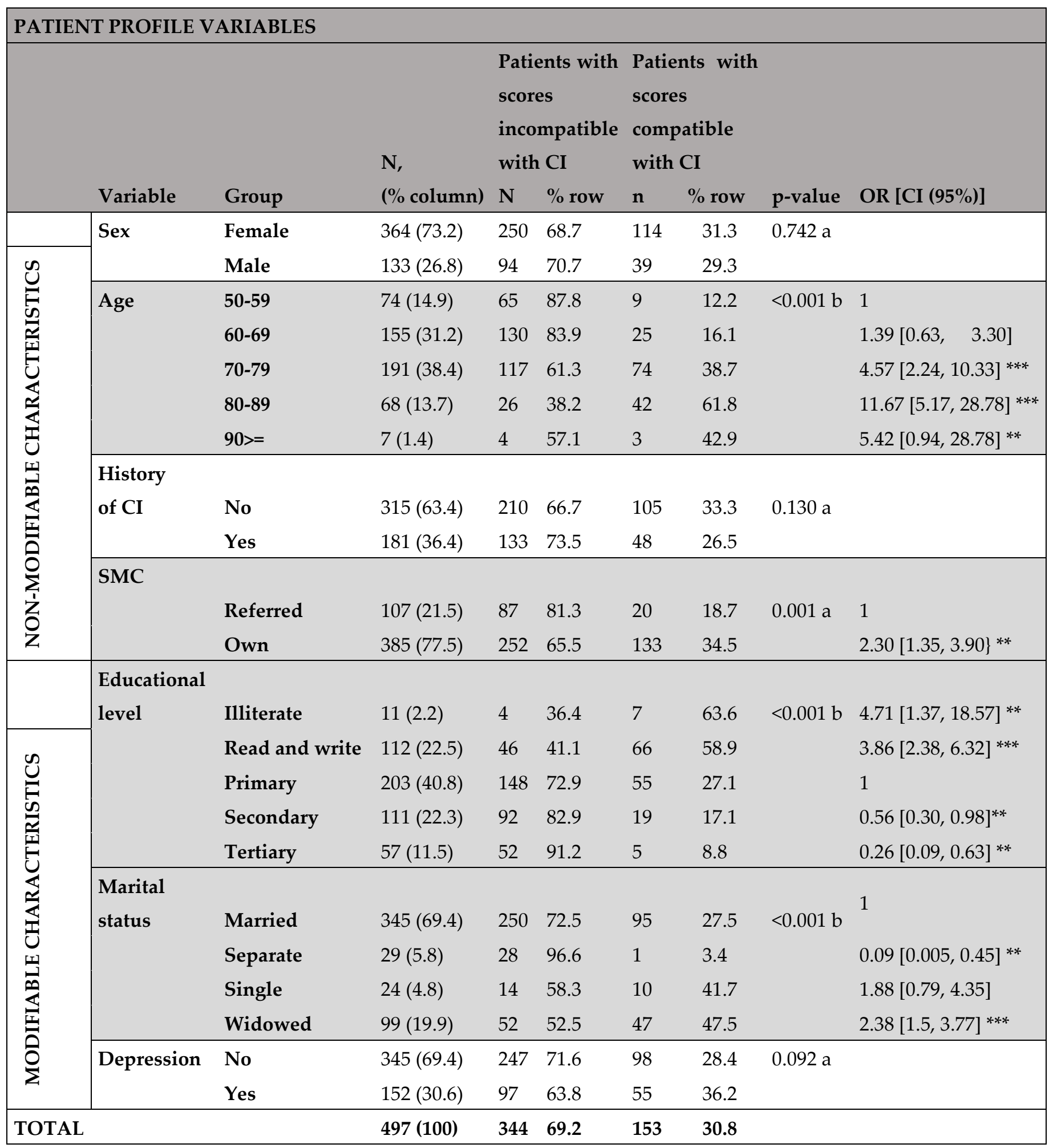

3.3. Cognitive reserve and cognitive stimulation. 
A higher level of education has a protective effect. Having higher education (greater cognitive reserve) decreases the odds of scores consistent with CI by 0.09 to 0.63 times that of primary school. That is a reduction of between $37 \%$ and $91 \%$ of the reference odds. On the other hand, marital status may also explain scores compatible with suffering CI. For example, being widowed (generally more socially isolated patients) may increase the odds of married patients by between 1.5 and 3.77 times.

Table 3: Association between obtaining or not obtaining scores compatible with $\mathrm{CI}$ and the quantitative variables on living and intellectual habits. $\mathrm{T}$ test for independent samples (univariate logistic models: ${ }^{*}: \mathrm{p}$-value $<0.1{ }^{* * *}$ : $\mathrm{p}$-value $<0.05 ;{ }^{* * *}: \mathrm{p}$ value< 0.001$)$. CI: cognitive impairment.

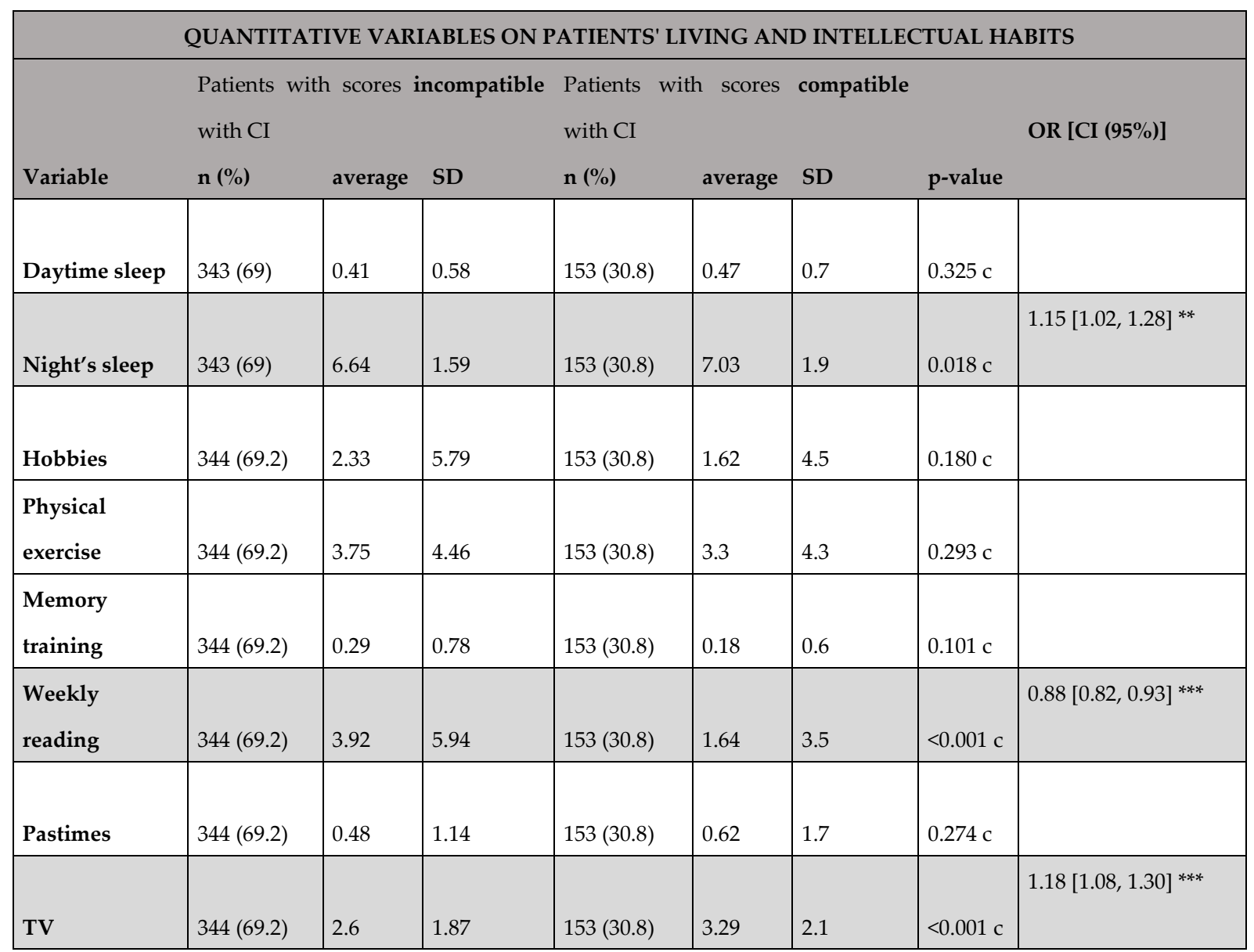

As table 3 shows, each hour of nightly sleep can increase the odds of obtaining scores compatible with CI by between 1.02 and 1.28 times. Similar to the increase for each additional hour of daily television, between 1.08 and 1.30 times. However, each hour of reading per week can decrease the odds by 0.82 to 0.93 times; that is, an odds reduction of between $7 \%$ and $18 \%$. With the sample we have available, we have not been able to determine any association with the time patients sleep during the day, nor with time spent on hobbies, exercise, memory training or pastimes.

Given the significant associations observed in Table 3, it has been analyzed whether the frequency (equal, more or less than before) with which they practice each of these habits, can explain that patients obtain scores compatible with CI (Table 4). 
Table 4: Association between compatible scores for $\mathrm{CI}$ and qualitative variables on living and intellectual habits (univariate logistic models: *: p-value< $0.1{ }^{* *}$ : p-value $<0.05 ;{ }^{* * *}$ : p-value< 0.001$)$. CI: cognitive impairment.

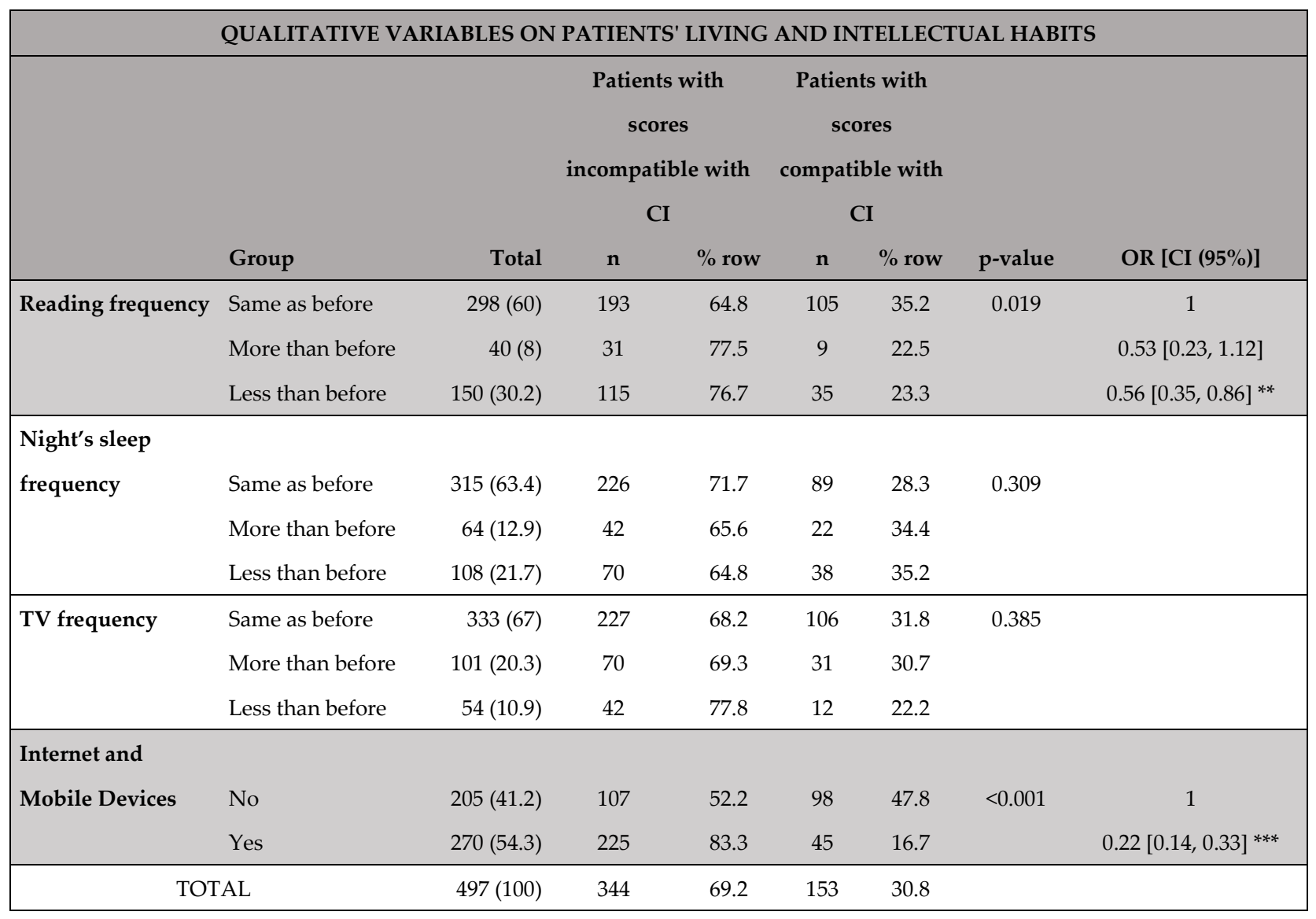

Initially, it seems paradoxical that the hours of reading have a protective effect and that, "reading less than before" also has a protective effect with respect to the reference category ("reading as before"). However, further analysis of the association between reading frequency and use of the Internet and mobile devices shows that of the 150 patients who say they read less than before, 93 are Internet users (63.3\%), 54 do not use it (36.7\%) and in 3 of them this data is unknown. This percentage is the highest of the three categories: "read the same as before", "read more than before" or "read less than before". For this reason, it seems that patients, who read less than before, have substituted that reading time by the consumption of Internet and mobile devices.

In addition, the use of the Internet and mobile devices is a habit related to our variable of interest, whose OR can range from 0.14 to 0.33 . Therefore, this habit can produce a decrease in the odds of obtaining scores compatible with $\mathrm{CI}$ with respect to non-user patients of between $67 \%$ and $86 \%$. From this preliminary analysis, we deduce that there are eight characteristics associated with obtaining scores compatible with $\mathrm{CI}$, related to the patient's profile and life habits. In addition, the associations between these eight characteristics have been analyzed. All this has been schematized in Figure 2, using an adaptation of the Ishikawa diagram. 


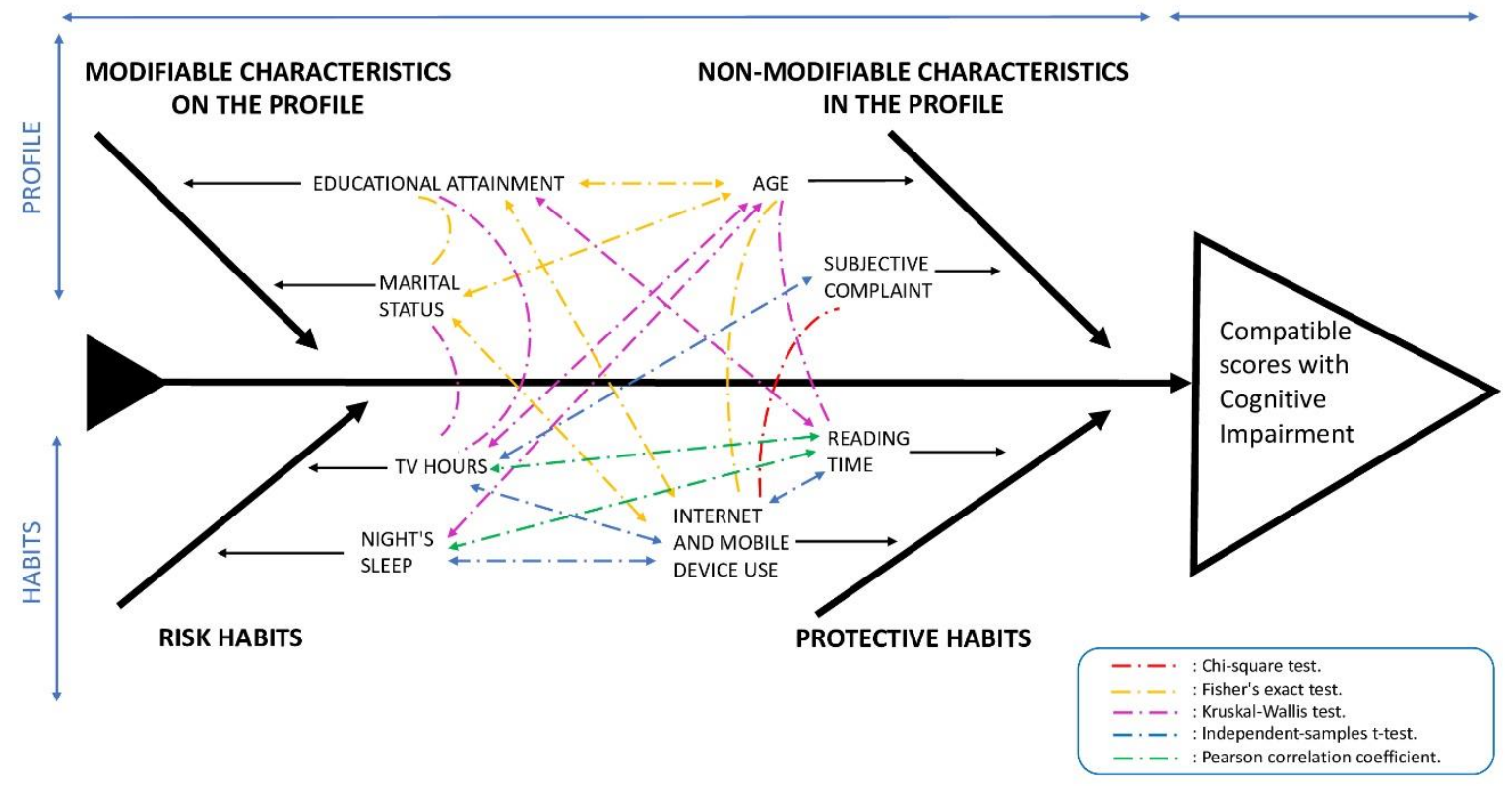

Figure 2: Characteristics associated with obtaining scores compatible with cognitive impairment (continuous arrows) and statistically significant associations between these characteristics (discontinuous arrows).

Table 5 and Figure 3 include the estimation of the OR of different multivariate logistic regressions, evaluated with $95 \%$ confidence. The first model corresponds to a logistic regression with the variables on the profile of the patients that have been associated with the variable of interest only. As can be seen, the four variables maintain their significance obtained in the univariate models, shown in Table 2.

However, the categories of the marital status variable differ in significance in relation to the univariate model. That is, adjusting for age, SMC and level of studies, it is observed that being single (patients with greater social isolation generally) can increase the odds of married patients by between 1.57 and 12.1 times. Moreover, adjusting for SMC, educational level and marital status, the age range of 80-89 years is the feature that can increase the odds of the youngest, between 2.03 and 13.50 times. 

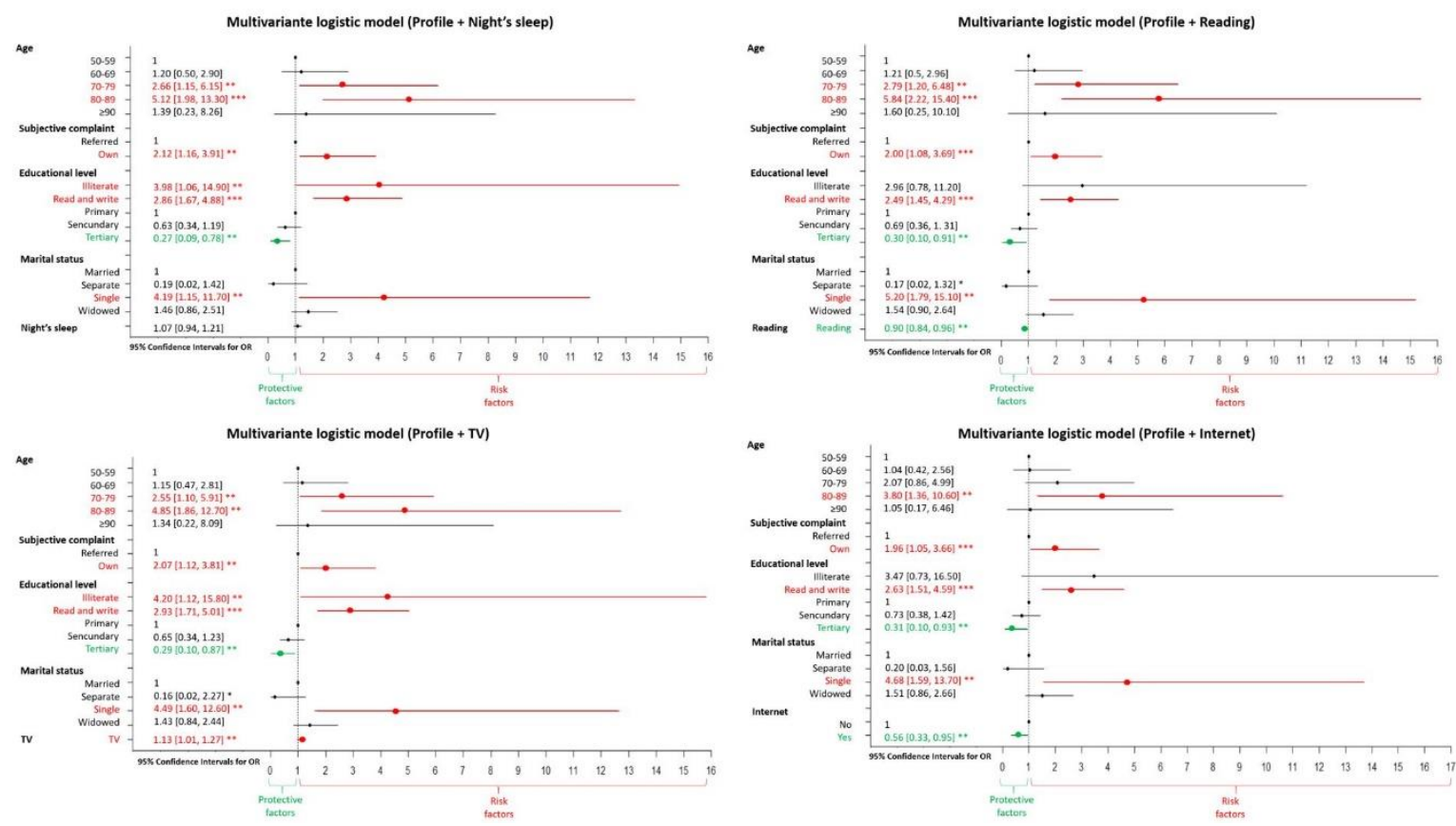

Figure 3confidence intervals: (95\%) for OR for the patient's profile and each of the significant lifestyle habits $\left({ }^{*}: \mathrm{p}\right.$ value $<0.1$; ${ }^{* *}$ : $\mathrm{p}$ value $<0.05 ; * *$ : $\mathrm{p}$ value $\left.<0.001\right)$.

Table 5: Multivariate logistic regression models on the patient profile and each of the significant life habits $\left({ }^{*}\right.$ : $p$-value $<0.1 ;{ }^{* *}$ : p-value< $0.05 ; * * *$ p-value $<0.001)$.

\begin{tabular}{|c|c|c|c|c|c|c|}
\hline & \multicolumn{5}{|c|}{ MULTIVARIATE LOGISTICS MODELS } \\
\hline & & $\begin{array}{c}\text { Profile } \\
\text { OR [CI (95\%)] }\end{array}$ & $\begin{array}{c}\text { Profile + Night's } \\
\text { sleep } \\
\text { OR [CI }(95 \%)]\end{array}$ & OR [CI (95\%)] & 0R [CI (95\%)] & $\begin{array}{c}\text { Profile + Internet } \\
\text { OR [CI (95\%)] }\end{array}$ \\
\hline \multirow{15}{*}{ 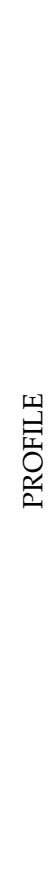 } & Age & & & & & \\
\hline & $50-59$ & 1 & 1 & 1 & 1 & 1 \\
\hline & $60-69$ & $1.17[0.49, \quad 2.84]$ & $1.20[0.50, \quad 2.90]$ & $1.21[0.50, \quad 2.96]$ & $1.15[0.47, \quad 2.81]$ & $1.04[0.42, \quad 2.56]$ \\
\hline & $70-79$ & $2.67[1.16,6.16]^{* *}$ & $2.66[1.15,6.15]^{* *}$ & $2.79[1.20,6.48]^{* *}$ & $2.55[1.10,5.91]^{* *}$ & $2.07[0.86,4.99]$ \\
\hline & $80-89$ & $5.24[2.03,13.50]^{* * *}$ & $5.12[1.98,13.30]^{* * *}$ & $5.84[2.22,15.40]^{* * *}$ & $4.85[1.86,12.70]^{* *}$ & $3.80[1.36,10.60]$ ** \\
\hline & $90>=$ & $1.41[0.24,8.46]$ & $1.39[0.23,8.26]$ & $1.60[0.25,10.10]$ & $1.34[0.22,8.09]$ & $1.05[0.17,6.46]$ \\
\hline & SMC & & & & & \\
\hline & Referred & 1 & 1 & 1 & 1 & 1 \\
\hline & Own & $2.12[1.16,3.90]^{* *}$ & $2.12[1.16,3.91]^{* *}$ & $2.0[1.08,3.69]^{* *}$ & $2.07[1.12,3.81]^{* *}$ & $1.96[1.05,3.66]^{* *}$ \\
\hline & Educational & & & & & \\
\hline & level & $3.93[1.05,14.7]^{* *}$ & $3.98[1.06,14.90]^{* *}$ & $2.96[0.78,11.20]$ & $4.20[1.12,15.80]^{* *}$ & $3.47[0.73,16.50]$ \\
\hline & Illiterate & $2.83[1.66,4.82]^{* * *}$ & $2.86[1.67,4.88]^{* * *}$ & $2.49[1.45,4.29]^{* * *}$ & $2.93[1.71,5.01]^{* * *}$ & $2.63[1.51,4.59]^{* * *}$ \\
\hline & Read and write & 1 & 1 & 1 & 1 & 1 \\
\hline & Primary & $0.60[0.32,1.14]$ & $0.63[0.34,1.19]$ & $0.69[0.36,1.31]$ & $0.65[0.34,1.23]$ & $0.73[0.38,1.42]$ \\
\hline & Secundary & $0.26[0.09,0.76]^{* *}$ & $0.27[0.09,0.78]^{* *}$ & $0.30[0.10,0.91]^{* *}$ & $0.29[0.10,0.87]^{* *}$ & $0.31[0.10,0.93]^{* *}$ \\
\hline
\end{tabular}




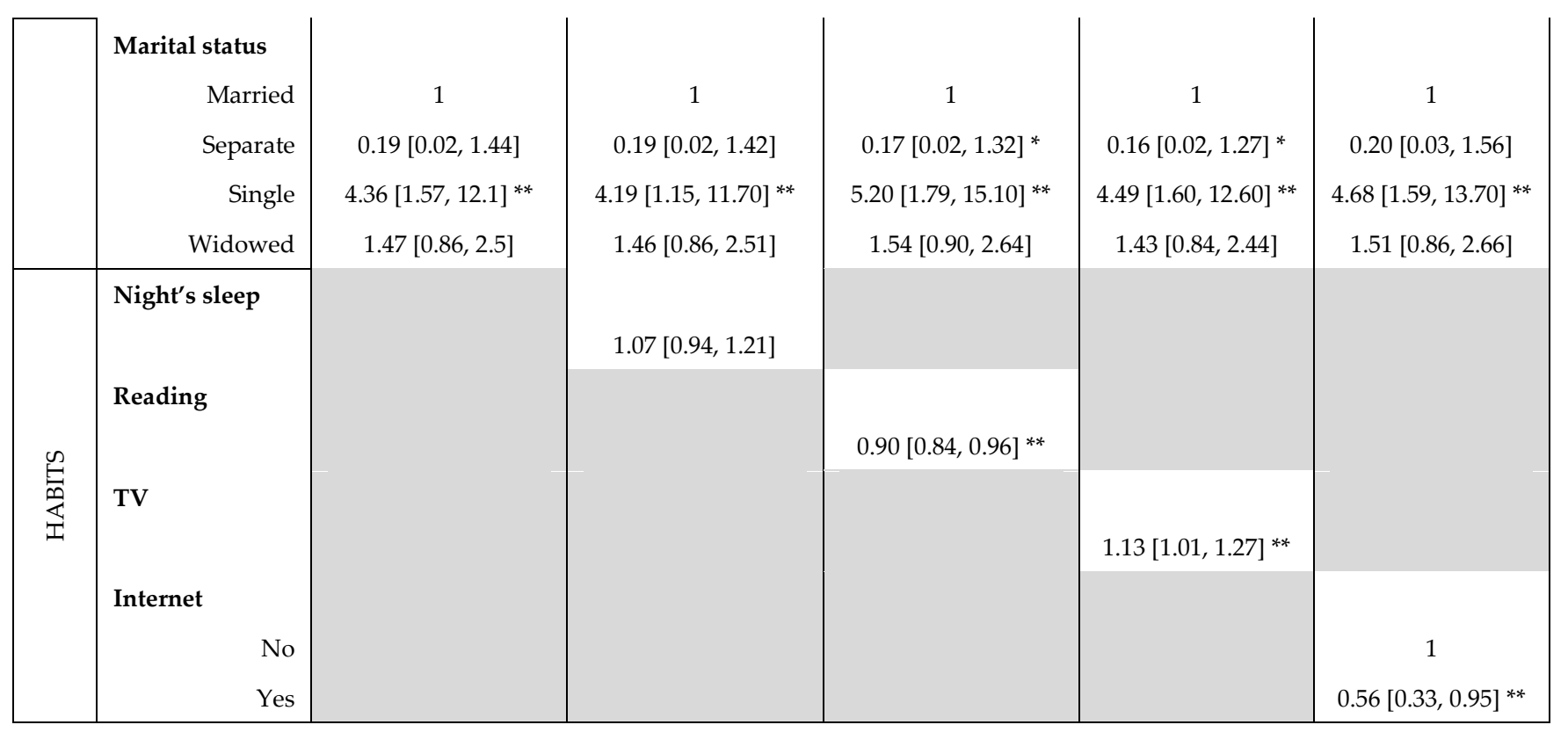

\subsection{Multivariate logistic regression models on the patient profile and each of the significant modifiable life habits.}

The next models analyze the effect of significant habits separately from Tables 3 and 4, when adjusted for the four traits of the patient profile. As can be seen in the second model in Table 5 and the first bullet in Figure 3, the hours of sleep at night lose their significance with respect to the probability of obtaining scores compatible with CI. In the third model, the reading habit maintains its significant protective effect. For every hour of reading per week, the odds of obtaining scores compatible with CI are expected to be reduced by between $4 \%$ and $16 \%$.

In the fourth model, the hours of TV watching maintain their risk effect, so that each hour of daily TV can raise the odds by between $1 \%$ and $27 \%$. Finally, in the fifth model, the use of Internet and mobile devices maintains its protective effect, which can reduce the odds of non-users by between $5 \%$ and $67 \%$. It should be noted that, in models 3,4 and 5 , the estimation by confidence intervals of the OR for each habitat is practically stable with respect to those obtained in univariate models. In addition, the variables on the profile also maintain their effects stable in each of the multivariate models.

\section{Discussion}

The present work belongs to the research line of the CRIDECO Team [14-16] and aims to articulate a protocol that favours the early detection of CI by neurological diagnosis. Alzheimer disease is typically a disease of old age. Our results estimated that being older than 70 years old can increase the odds of obtaining scores compatible with suffering CI from younger patients between 2.24 and 10.33 times. 
One of the strengths of the study resides in analyzing the influence that qualitative and quantitative variables scores compatible with $\mathrm{CI}$ in any of the tests used on a population older than 50 years, with subjective memory complaint.

Thirty percent of patients with subjective memory complaints have obtained scores compatible with having CI. This percentage is in keeping with other studies suggesting the idea that subjective cognitive complaints has been considered as an early clinical marker of pathology and help further understanding from predementia stages $[5,17,18]$.

In a meta-analysis of longitudinal studies, it was observed that in patients with SCD, (with a follow-up of at least 4 years), the evolution to dementia occurred in $14 \%$ and to $\mathrm{MCI}$ in $27 \%$ [4]. Subjective complaint of own memory represents $77.5 \%$ of our population, which is statistically related to the female sex, feeling depressed and benzodiazepines consumption. This could reveal a percentage even higher, of future neurodegenerative diseases being aware that these patients fulfilled not only the self-experience in cognitive decline (as corresponds with the SCD term) but also a score compatible with CI. Referred complaint, not being aware of memory problems (22.5), is significantly more frequent in men, in patients who do not feel depressed or in patients who do not take benzodiazepines.

Moreover, having own subjective complaint can almost quadruple the odds of obtaining CIcompatible scores for patients who are not aware of having a memory problem. Not being aware of memory problems predicts onset of Alzheimer's disease [19], this highlights the importance of monitoring cognitive decline in both cases conscious and amnestic patients.

Marital status may also explain the scores consistent with having CI in our study. Being a widower can increase the odds of married patients between 1.5 and 3.77 times. Adjusting for age, SMC and educational level, it is observed that being single can increase the odds of married patients between 1.57 and 12.1 times. In general, old widows and old singles presenting a greater social isolation. These results are supported by other studies that affirm that social isolation of those living alone may be at risk for decline cognitive function and marital status have the potential to buffer the effects of low mood on dementia onset $[8,20,21,22]$. Cognitive inactivity due to isolation may explain the impact of marital status on CI, or perhaps feelings of impaired cognition make patients to avoid social contact [1].

The cognitive reserve (through and cognitive stimulation, in both intellectual and leisure activities, shows protection against CI. In fact, not having reached secondary studies is a predictor of $\mathrm{CI}$ [1]. In the present work, we confirm that having higher studies (higher than primary studies) decrease the odds of obtaining CI-compatible scores between $37 \%$ and $91 \%$.

Lifestyle factors and cognitive stimulation have been found to improve cognition and determining individual risk of dementia [23]. We find four lifestyle habits associated with obtaining scores compatible with CI: sleep hours, watching TV, reading time and use of internet. The results 
for those users described as internet consumers (57-86\%) and usual readers (7-18\%) show a score considered protective towards CI. Nevertheless, watching TV (8-30\%) and additional hours of sleep (2-28\%) have also been related to scores compatible with CI. These results coincide with what has been described in the literature in relation to extra diurnal and nocturnal sleep [24], the use of internet $[10,25,26]$, reading habits [27] and television consumption [28,29].

Therefore, our results confirm, what is reflected in a review, in which leisure activities involving brain stimulation such us: use of internet, boarding games and reading, are shown as protective to CI [30]. Moreover, it was proved that reading increases our brain connectivity in areas related to language and sensory region [31]. Conversely, long hours in front of the television, favours the frontal lobe to become thicker, causing a decrease in verbal reasoning ability [32].

The explanation could be that the way in which we interact with books and television is very different. While television is designed to be more passive, we just sit back and receive information without having to make great efforts, whereas books require an amount of concentration and reflection.

Moreover, the use of the internet through mobile devices, smart phones (games, internet search) can increase cognitive stimulation to the point of reducing age-related cognitive decline [10].

In the present study, we were surprised to find that the variable "reading less than before" appears to be initially protective (4.8-63.7\%), in the same way as was the use of the internet. Trying to justify this result, we discovered that $63 \%$ of those patients $(n=147)$ that have been revealed as "reduced reading habit" are internet users. According to this, the reading habit could be substituted by the internet habit, which explains the protective effect exerted by this variable ("Reading less").

With the aim to clarify this controversial result, we adjusted our results by patient profile (age, educational level, subjective memory complaint and marital status), and, therefore, it was the one variable "reading more than before" that was responsible for the statistically significant protection (6.3-85.4\%). Because of this adjustment, the variable "use of internet" still being significant in its protective effect. Conversely, for the variable "additional hours of sleep ", the statistically protective effect disappears. This result appears to be contrary to bibliography. Thus, the association between an extra nocturnal sleep and a compatible score with cognitive deterioration for patients with subjetive memory complaint may depend on their own profile (marital status, educational level, age) and not exclusively on the additional hours of sleep. This evidence has shown in a review in which variables such as age, previous pathologies, polypharmacy, are defined as factors associated with cognitive decline [33].

Adjustment for individual profiles was stablished with the objective of estimating the "magnitude" of those variables (reading habits, use of internet, TV consumption) statistically associated with (compatible or incompatible) scores with cognitive impairment, Consequently, on the one hand "one hour of reading per week" is able to reduce the odds of positive CI scores in 4- 
$16 \%$. Along the same line, internet use reduces the odds of positive CI scores in $5-67 \%$. On the other hand, one extra hour of TV per week increases the risk of compatible scores with CI in 1-27\%. The effect of extra nocturnal sleep was not evaluated because of the lack of significance when adjusting by profile.

It is surprising that the effect of the internet in our sample varies in a range from $5-67 \%$, which may be because we have not registered quantitatively what the term "use of internet" is referred to. In fact, we recognized this point as one of the most important limitations of our study.

In the presence of reading, the variable "use of internet" loses its significance. That is, for a patient with a reading habit and internet consumption, "reading" is the variable mainly responsible of the protective effect in the odds reduction in terms of score compatible with CI. In the same way, the habit of reading nullifies the statistically significance predisposing effect of TV consumption with scores compatible with $\mathrm{CI}$ in at least one of the tests used for the present study.

In conclusion, reading, (from the variables we studied), can be considered, the variable that exerts the highest influence in the cognitive maintenance of our sample of study. Accordingly, reading would be the most interesting item to take into account when developing programs to stimulate cognition in the elderly. Reading is even able to supersede the supposed effect of cognitive decline related to TV consumption.

Among the variables that in Figure 2 reveal a statistically significant association, it is important to highlight that the older the patient, the greater the number of hours of TV consumption, and hours of sleep and the lower the use of the Internet, and the habit of reading. This could explain in older adults the increased risk of CI, not just related with age but with lifestyles that do not stimulate brain activity. Likewise, the presence of own subjective complaint (statistically associated with feeling depressed and the consumption of benzodiazepines) is associated with patients with a greater habit of TV consumption and less use of the Internet.

This discovery elucidates the importance of designing programs for older adults that include brain stimulation. Thus, stimulating the habit of reading and the use of the Internet in the population at risk or with a declared diagnosis of $\mathrm{CI}$ in the earliest stages, in order to slow down the evolution of the disease. It is also important to point out that a self-experience of memory complaint must be individually considered in our healthcare system.

Our future lines of work pretend to elucidate the relationship between compatible or incompatible scores with a definitive neurological diagnosis, in order to develop effective cognitive training programs.

\section{Conclusions}

The presence of own subjective memory complaint is statistically associated with a score compatible with CI, which is more typical for the female sex and feeling depressed, as well as 
benzodiazepines consumption. Older adults are especially at risk of CI because of their lifestyle habits. Internet use has been proved to be a protective character, as well as reading exert in a significant reduction of the odds of compatible scores with CI. Future studies are needed to ensure a real association between a compatible score and neurological diagnosis of CI. Along with a detailed analysis of internet consumption, that could let us adjust the specific protection range of this variable according to individual profile.

Author Contributions: Conceptualization, LM and RS.; methodology, LM, HR and RS.; software, HR and MA.; validation, MG and MA.; formal analysis, MA.; investigation, HR and RS.; resources, MG.; data curation, HR.; writing - original draft preparation, LM, MG, MA.; writing-review and editing, LM.; visualization, RS, MG.; supervision RS, LM; project administration, LM.; funding acquisition, LM. All authors listed have made a substantial and direct contribution to the work and have read and agreed to the published version of the manuscript.

Acknowledgments: We want to acknowledge to all pharmacy's participants in the study, to all the general practitioners who collaborated in the diagnosis and to Cátedra DeCo Micof-UCH that have funded part of the research. In addition, to all the patients who trusted us and participated in our study.

Conflicts of Interest: The authors declare no conflict of interest.

\section{References}

1. Livingston, G.; Sommerlad, A.; Orgeta, V.; Costafreda, S.G.; Huntley, J.; Ames, D.; Ballard, C.; Banerjee, S.; Burns, A.; Cohen-Mansfield, J.; et al. Dementia prevention, intervention, and care. Lancet, 2017, 16, 26732734. doi: 10.1016/S0140-6736(17)31363-6.

2. Alacreu, M.; Pardo, J.; Azorín, M.; Climent, M.T.; Gasull, V.; Moreno, L. Importance of increasing modifiable risk factors knowledge on Alzheimer's disease among community pharmacists and general practitioners in Spain O Front. Pharmacol. 2019, 14, 860. doi: 10.3389/fphar.2019.00860. eCollection 2019.

3. Lara, E.; Koyanagi, A.; Olaya, B.; Lobo, A.; Miret, M.; Tyrovolas, S.; Ayuso-Mateos, J.L.; Haro, J.M. Mild cognitive impairment in a Spanish representative sample: prevalence and associated factors. Int. J. Geriatr. Psychiatry, 2016, 31, 858-867. doi: 10.1002/gps.4398.

4. Jessen, F.; Amariglio R.E.; Buckley R.F.; van der Flier W.M.; Han Y.; Molinuevo J.L.; Rabin, L.; Rentz, D.M.; Rodriguez-Gómez, O.; Saykin, A.J.; et al. The characterization of subjective cognitive decline. Lancet Neurol. 2020, 19, 271-278. doi: 10.1016/S1474-4422(19)30368-0.

5. Mitchell, A.J.; Beaumont, H.; Ferguson, D.; Yadegarfar, M.; Stubbs, B. Risk of dementia and mild cognitive impairment in older people with subjective memory complaints: meta-analysis. Acta Psychiatr. Scand. 2014, 130, 439-451. doi:10.1111/acps.12336.

6. Stern, Y. Cognitive reserve in ageing and Alzheimer's disease. Lancet Neurol. 2012, 11, 1006-1012. doi:10.1016/S1474-4422(12)70191-6.

7. Jones, R.N.; Manly, J., Glymour, M.M.; Rentz D.M.; Jefferson A.L.; Stern, Y. Conceptual and measurement challenges in research on cognitive, reserve. J. Int. Neuropsychol. Soc. 2011, 17, 593-601. doi: 10.1017/S1355617710001748.

8. Evans, I.E.M.; Martyr, A.; Collins, R.; Brayne, C.; Clare, L. Social Isolation and Cognitive Function in Later Life: A Systematic Review and Meta-Analysis. J. Alzheimers Dis. 2019, 70, S119-S144. doi:10.3233/JAD180501.

9. Gindrat, A.D., Chytiris, M.; Balerna, M. Rouiller, E.M.; Ghosh, A. Use-dependent cortical processing from fingertips in touchscreen phone users. Curr. Biol. 2015, 25, 109-116. doi: 10.1016/j.cub.2014.11.026.

10. Firth, J.; Torous, J.; Stubbs, B.; Firth, J.A.; Steiner, G.Z.; Smith, L.; Alvarez-Jimenez, A.; Gleeson, J.; Vancampfort, D.; Armitage C.J., et al. The "online brain": how the Internet may be changing our cognition. World Psychiatry, 2019, 18, 119-129. doi: 10.1002/wps.20617. 
11. Böhm, P.; Peña-Casanova, J.; Gramunt, N.; Manero, R.M.; Terrón, C.; Quiñones-Ubeda, S. Spanish version of the Memory Impairment Screen (MIS): normative data and discriminant validity. Neurologia 2005, 20, 402-411.

12. Martínez de la Iglesia, J.; Dueñas Herrero, R.; Onís Vilches, M.C.; Aguado Taberné, C.; Albert Colomer, C.; Luque Luque, R. Adaptación y validación al castellano del cuestionario de Pfeiffer (SPMSQ) para detectar la existencia de deterioro cognitivo en personas mayores de 65 años. Medicina Clinica, 2001, 117, 129-134. doi.org/10.1016/S0025-7753(01)72040-4.

13. López Pérez-Díaz, A.G.; Calero, M.D.; Navarro-González, E. Predicción del deterioro cognitivo en ancianos mediante el análisis del rendimiento en fluidez verbal y en atención sostenida. Rev. Neurol. 2013, 56, 1-7. doi: https://doi.org/10.33588/rn.5601.2012281.

14. Moreno, L.; Climent, M.T.; Vilaplana, A.M.; Arnedo, A.; Vilar, J. () Life Styles associated cognitive impairment. Study from the community pharmacy. Rev. Inv. Clin. 2013, 65, 500-9

15. Climent, M.T.; Pardo, J.; Muñoz-Almaraz, F.J.; Guerrero, M.D.; Moreno, L. Decision Tree for Early Detection of Cognitive Impairment by Community Pharmacists. Front. Pharmacol. 2018, 9, 1232. doi:10.3389/fphar.2018.01232

16. Muñoz-Almaraz, F.J.; Climent, M.T.; Guerrero, M.D.; Moreno, L.; Pardo, J.A. Machine Learning Approach to Design an Efficient Selective Screening of Mild Cognitive Impairment. J. Vis. Exp. 2020, 11,155. doi: $10.3791 / 59649$

17. Neto, S.A.; Nitrini, R.D. Subjective cognitive decline: The first clinical manifestation of Alzheimer's disease? Dement. Neuropsychol. 2016, 10,170-177. doi: 10.1590/S1980-5764-2016DN1003002.

18. Arvanitakis, Z.; Leurgans, S.E.; Fleischman, D.A.; Schneider, J.A.; Rajan, K.B.; Pruzin, J.J.; Shah, R.C.; Evans, D.A.; Barnes, L.L.; Bennett, D.A. Memory complaints, dementia, and neuropathology in older blacks and whites. Ann. Neurol. 2018, 83, 718-729. doi:10.1002/ana.25189.

19. Therriault, J. Ng, K.P.; Pascoal, T.A.; Mathotaarachchi, S.; Kang, M.S.; Struyfs, H.; Shin, M.; Benedet, A.L.; Walpola, I.C-; Nair, V.; et al. Anosognosia predicts default mode network hypometabolism and clinical progression to dementia. Neurology, 2018, 90, e932-e939. doi: 10.1212/WNL.0000000000005120.

20. Sundström A.; Westerlund O.; Kotyrlo E. Marital status and risk of dementia: a nationwide populationbased prospective study from Sweden. B.M.J. Open. 2016, 6, e008565. doi:10.1136/bmjopen-2015-008565.

21. Sjöberg L.; Fratiglioni, L.; Lövdén, M.; Wang, H.X. Low Mood and Risk of Dementia: The Role of, Marital Status and Living Situation. Am. J. Geriatr. Psychiatry. 2020, 28, 33-44. doi: 10.1016/j.jagp.2019.08.014

22. Sommerlad, A.; Ruegger, J.; Singh-Manoux, A.; Lewis, G.; Livingston, G. Marriage and risk of dementia: systematic review and meta-analysis of observational studies. J. Neurol. Neurosurg. Psychiatry. 2018, 89, 231238. doi: 10.1136/jnnp-2017-316274.

23. Mintzer, J.; Donovan, K.A.; Kindy, A.Z.; Lock, S.L.; Chura, L.R.; Barracca, N. Lifestyle Choices and Brain Health. Front. Med. 2019, 6, 204. doi:10.3389/fmed.2019.00204

24. Gabelle, A.; Gutierrez, L.A.; Jaussent, I.; Navucet, S.; Grasselli, C.; Bennys, K.; Marelli, C.; David, R.; Andrieu, S.; Berr, C.; et al. Excessive Sleepiness and Longer Nighttime in Bed Increase the Risk of Cognitive Decline in Frail Elderly Subjects: The MAPT-Sleep. Study. Front. Aging Neurosci. 2017, 9, 312. doi:10.3389/fnagi.2017.00312.

25. d'Orsi, E.; Xavier, A.J.; Rafnsson, S.B.; Steptoe, A.; Hogervorst, E.; Orrell, M. Is use of the Internet in midlife associated with lower dementia incidence? Results from the English Longitudinal Study of Ageing. Aging Ment. Health. 2018, 22, 1525-1533. doi:10.1080/13607863.2017.1360840.

26. Krug, R.R.; d'Orsi, E.; Xavier, A.J. Association between use of Internet and the cognitive function in older adults, populational longitudinal study EpiFloripa Idoso. Associação entre o uso de Internet e a função cognitiva de idosos, estudo longitudinal populacional Epifloripa Idoso. Rev. Bras. Epidemiol. 2019, 22, e190012. doi:10.1590/1980-549720190012.

27. Juncos-Rabadán, O.; Pereiro, A.X.; Facal, D.; Lojo, C.; Caamaño, J.A.; Sueiro, J.; Bóveda, J.; Eiroa, P. Prevalence and correlates of mild cognitive impairment in adults aged over 50 years with subjective cognitive complaints in primary care centers. Geriatr. Gerontol. Int. 2014, 14, 667-673. doi:10.1111/ggi.12157

28. Fogel, J.; Carlson, M.C. Soap operas and talk shows on television are associated with poorer cognition in older women. South Med. J. 2006, 99, 226-233. doi: 10.1097/01.smj.0000198270.52240.93.

29. Lindstrom, H.A.; Fritsch, T.; Petot, G.; Smyth, K.A.; Chen, C.H.; Debanne, S.M. Lerner, A. J.; Friedland, R.P. The relationships between television viewing in midlife and the development of Alzheimer's disease in a case-control study. Brain and Cognition, 2005, 58, 157-165. https://doi.org/10.1016/j.bandc.2004.09.020 
30. Iizuka, A.; Suzuki, H.; Ogawa, S.; Kobayashi-Cuya., K.E.; Kobayashi, M.; Takebayashi, T.; Fujiwara, Y. Can cognitive leisure activity prevent cognitive decline in older adults? A systematic review of intervention studies. Geriatr. Gerontol. Int. 2019, 19, 469-482. doi:10.1111/ggi.13671.

31. Berns, G.S.: Blaine, K.; Prietula J.M.; Pye, B.E. Short- and long-term effects of a novel on nonnectivity in the brain. Brain Connectivity, 2013, 3, 590-600. doi.org/10.1089/brain.2013.0166

32. Takeuchi, H.; Taki, Y.; Hashizume, H.; Asano, K.; Asano, M.; Sassa, Y.; Yokota, S.; Kotozaki, Y,; Nouchi, R.; Kawashima, R. The Impact of Television Viewing on Brain Structures: Cross-Sectional and Longitudinal Analyses. Cerebral Cortex, 2015, 25, 1188-1197, https://doi.org/10.1093/cercor/bht315.

33. Wennberg, A.M.V.; Wu, M.N.; Rosenberg, P.B.; Spira, A.P. Sleep Disturbance, Cognitive Decline, and Dementia: A Review. Semin Neurol. 2017, 37, 395-406. doi:10.1055/s-0037-1604351 